\title{
Effect of Neutral and Acidic Oligosaccharides on Fecal IL-8 and Fecal Calprotectin in Preterm Infants
}

\author{
ELISABETH A. M. WESTERBEEK, ERIK MØRCH, HARRIE N. LAFEBER, WILLEM P. F. FETTER, JOS W. R. TWISK, \\ AND RUURD M. VAN ELBURG \\ Department of Pediatrics [E.A.M.W., E.M., H.N.L., W.P.F.F., R.M.E.], Institute of Research in Extramural Medicine [J.W.R.T.], VU \\ University Medical Center, De Boelelaan 1117, 1081 HV Amsterdam, The Netherlands
}

\begin{abstract}
The gastrointestinal inflammatory response may play a role in the susceptibility of preterm infants for infections. We previously reported a trend toward lower endogenous infection morbidity after enteral supplementation of neutral and acidic oligosaccharides $\left({ }_{\mathrm{SC}} \mathrm{GOS} /{ }_{\mathrm{LC}} \mathrm{FOS} / \mathrm{AOS}\right)$. We hypothesize that enteral supplementation of prebiotics may decrease infectious morbidity by reducing intestinal inflammation. Therefore, we aimed to determine the effect of enteral supplementation of prebiotics on intestinal inflammation, as measured by fecal IL-8 (f-IL-8) and calprotectin (f-calprotectin), in preterm infants. In a randomized controlled trial, infants with a GA $<32 \mathrm{wk}$ and/or birth weight $<1,500 \mathrm{~g}$ received enteral supplementation of prebiotics or placebo (maltodextrin) between d 3 and 30 of life. F-IL-8 and f-calprotectin was assessed at baseline, d 7, 14, and 30 of life. In total, 113 infants were included. Baseline patient and nutritional characteristics were not different in the ${ }_{\mathrm{SC}} \mathrm{GOS} /{ }_{\mathrm{LC}} \mathrm{FOS} / \mathrm{AOS}(n=55)$ and the placebo group $(n=58)$. Enteral supplementation of prebiotics had no effect on f-IL-8 and f-calprotectin. F-IL-8 and f-calprotectin were strongly correlated at all time points $(p<0.001)$. In conclusion, enteral supplementation of prebiotics ( $\mathrm{sC}_{\mathrm{GOS}} / \mathrm{LC}$ FOS/AOS) does not affect f-IL-8 and fcalprotectin levels in preterm infants. (Pediatr Res 69: 255-258, 2011)
\end{abstract}

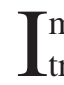
mmaturity of the immune system and the gastrointestinal tract may play a pivotal role in the susceptibility of preterm infants for infections. The maturation of the immune system is influenced by bacterial antigens of the intestinal microbiota (1). This interaction leads to metabolic and immunologic reactions by the epithelial cells and the underlying lymphoid cells and is called the bacterial-epithelial "cross talk" (2). The bacterialepithelial "cross talk" between the intestinal microbiota and immune system may cause an inflammatory response, which may further increase the susceptibility of preterm infants for serious infections and necrotizing enterocolitis (NEC) (3).

Invasive measurement of the inflammatory response of the gastrointestinal tract is difficult in preterm infants. However, noninvasive markers measuring inflammation of the gastrointestinal tract have been investigated. IL- 8 is a proinflammatory

Received April 7, 2010; accepted October 6, 2010.

Correspondence: Ruurd M. van Elburg, M.D., Ph.D., Department of Pediatrics, Division of Neonatology, VU University Medical Center, De Boelelaan 1117, 1081 HV Amsterdam, The Netherlands; e-mail: rm.vanelburg@vumc.nl

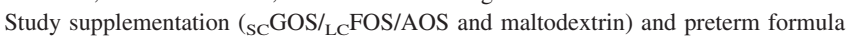
(Nenatal Start) and postdischarge formula (Nenatal 1) for this study was provided by Danone Research, Friedrichsdorf, Germany. The funding source was not involved in the analysis of the data or the interpretation of the results. This study is registered in the Controlled Clinical Trails registry (www.controlled-trials.com), number ISRCTN 16211826. cytokine produced by myelomonocytic cells that attracts and activates neutrophils and monocytes (4). In children with inflammatory bowel disease, IL-8 from organ-cultured lamina propria biopsies is related to inflammatory activity (5). Furthermore, in an in vitro study, Nanthakumar et al. (6) showed an enhanced IL-8 response of immature human enterocytes compared with mature enterocytes of older children. IL-8 precedes calprotectin in the inflammatory cascade. Calprotectin, released from neutrophils and monocytes, has several biological functions including fungicidal, antibacterial, and antiproliferative effects $(7,8)$. Both IL-8 and calprotectin can be measured in the feces. Fecal calprotectin (f-calprotectin) is increased in preterm infants compared with term infants and is increased during intestinal inflammation, especially in infants with NEC (9-13).

Feeding with breast milk affects the intestinal colonization, bacterial-epithelial "cross talk," and as a consequence, the intestinal inflammatory response, partially through the action of its oligosaccharides $(14,15)$. More than 200 human milk oligosaccharides have been identified with significant variability between individuals over time (16). Until now, controversial results have been reported on the effect of type of feeding on the level of f-calprotectin in preterm (17) and term infants $(13,18-20)$.

In a previous study, we found that a prebiotic mixture of neutral ( ${ }_{\mathrm{SC}} \mathrm{GOS} /{ }_{\mathrm{LC}} \mathrm{FOS}$ ) and acidic (AOS) oligosaccharides did not significantly decrease serious infectious morbidity in preterm infants. However, if ${ }_{\mathrm{SC}} \mathrm{GOS} /{ }_{\mathrm{LC}} \mathrm{FOS} / \mathrm{AOS}$ was given in sufficient amounts (per-protocol analysis), there was a significant decrease of serious endogenous infections in preterm infants (21). We hypothesize that prebiotics may prevent adherence of bacteria to the intestinal mucosa, thereby reducing the inflammatory response. This reduction in the inflammatory response, reflected by fecal IL-8 (f-IL-8) and fcalprotectin, may play a role in the previously found lower endogenous infection morbidity in preterm infants. The aims of this study were to determine the effect of enteral supplementation of prebiotics, ${ }_{S C} \mathrm{GOS} /{ }_{\mathrm{LC}} \mathrm{FOS} / \mathrm{AOS}$, on f-IL-8 and f-calprotectin in preterm infants and to determine host- and

Abbreviations: GEE, generalized estimating equations; ${ }_{\mathbf{S C}} \mathbf{G O S} / \mathbf{L C} \mathbf{F O S} /$ AOS, a mixture of neutral and acidic oligosaccharides; galacto-oligosaccharides/ fructo-oligosaccharides/acidic oligosaccharides; f-calprotectin, fecal calprotectin; f-IL-8, fecal IL-8; NEC, necrotizing entero colitis 
treatment-related factors associated with f-IL-8 and f-calprotectin in these preterm infants.

\section{METHODS}

Subjects. Infants with GA $<32$ wk and/or birth weight (BW) $<1,500 \mathrm{~g}$, admitted to the NICU of the VU University Medical Center, Amsterdam, were eligible for participation in the study. Exclusion criteria were infants with a GA $>34$ wk, major congenital or chromosomal anomalies, imminent death, and transfer to another hospital within $48 \mathrm{~h}$ after birth. The study protocol was approved by the medical ethical review board of the VU University Medical Center, Amsterdam, The Netherlands. Written informed consent was obtained from all parents.

Randomization, blinding, and treatment. After assignment to 1 of $3 \mathrm{BW}$ groups ( $\leq 799 \mathrm{~g}, 800-1,199 \mathrm{~g}, \geq 1,200 \mathrm{~g}$ ), the infants were randomly allocated to treatment within $48 \mathrm{~h}$ after birth to receive either enteral prebiotics, $72 \%{ }_{\mathrm{sC}} \mathrm{GOS} / 8 \%{ }_{\mathrm{LC}} \mathrm{FOS}$ and $20 \%$ AOS, or placebo powder (maltodextrin). An independent researcher used a computer-generated randomization table (provided by Danone Research, Friedrichsdorf, Germany) to assign infants to treatment with prebiotics or placebo. Investigators, parents, and medical and nursing staff were unaware of treatment allocation. The randomization code was broken after data analysis was performed. The prebiotics and the placebo powder (maltodextrin) were prepared and packed sterile under $\mathrm{N}_{2}$ environment in sachets (Danone Research). The two powders were indistinguishable by appearance, color, and smell. During the study period, prebiotics and placebo powder were monitored for stability and microbiological contamination. Supplementation with prebiotics or placebo was administered in increasing doses between $\mathrm{d} 3$ and 30 of life to a maximum of $1.5 \mathrm{~g} / \mathrm{kg} \cdot \mathrm{d}$ to breast milk or preterm formula. Two members of the nursing staff added the daily supplementation to breast milk or to preterm formula (Nenatal Start; Danone Research) according to the parents' choice. Per $100 \mathrm{~mL}$, the preterm formula provided $80 \mathrm{kcal}, 2.4 \mathrm{~g}$ protein (casein-whey protein ratio 40:60), $4.4 \mathrm{~g}$ fat, and $7.8 \mathrm{~g}$ carbohydrate. The preterm formula did not contain oligosaccharides.

Analysis of fecal calprotectin and fecal IL-8. For measurement of f-IL-8 and f-calprotectin, fecal samples were taken from the diaper with a sterile plastic spoon within $48 \mathrm{~h}$ after birth $(t=0)$, postnatal d $7(t=1), 14(t=2)$, and $30(t=3)$. Samples were stored in a sterile screw cap tube at $2-8^{\circ} \mathrm{C}$ for a maximum of $7 \mathrm{~d}$. Samples were extracted and diluted 1:50 with incubation buffer (in accordance with the manufacturer's instructions). The homogenate was micro centrifuged for $5 \mathrm{~min}$ at $10,000 \times \mathrm{g}$, and the supernatant was stored at $-20^{\circ} \mathrm{C}$ until analysis. IL-8 levels were measured by random-access chemiluminescence immunoassay using the Immulite 4000 4PL (Siemens Medical Solutions Diagnostics, Breda, The Netherlands). The random-access chemiluminescence immunoassay for measuring f-IL-8 has an intra-assay variation of $3 \%$ and an interassay variation of $13.6 \%$. IL-8 levels are expressed as picograms per milliliter feces.

Calprotectin levels were measured by ELISA using the Calprotectin-MPR8/14 kit (Buhlmann, Basel, Switzerland). Calprotectin levels are expressed in micrograms per gram feces. The ELISA for measuring f-calprotectin has an intra-assay variation of $4.7 \%$ and an interassay variation of $4.1 \%$.

Clinical and nutritional characteristics of the infants were assessed, including exclusive breast milk feeding, achievement of full enteral feeding $(>120$ $\mathrm{mL} / \mathrm{kg} / \mathrm{d}$ ), presence of a serious endogenous infection defined as sepsis, meningitis, pyelonephritis, pneumonia, or arthritis by a combination of clinical signs and a positive culture with bacteria other than Coagulase Negative Staphylococci $(21,22)$, and NEC (23).

Nutritional support. Protocol guidelines for the introduction of parenteral and enteral nutrition followed current practice at our NICU. Nutritional support was administered as previously described $(21,22)$. For each infant in the study, a feeding schedule was proposed based on BW and our guidelines. However, the ultimate responsibility for the administration of parenteral nutrition and advancement of enteral nutrition rested with the medical staff of our NICU or the responsible doctors in the regional hospitals. After discharge, all infants received breast milk or preterm formula (Nenatal Start ${ }^{\circledR}$ without oligosaccharides) and a postdischarge formula (Nenatal 1 without oligosaccharides) until the corrected age of 6 mo.

Statistical analysis. The sample size of 113 infants was based on the sample size calculation for the primary outcome of the main trial (serious infectious morbidity) (21). Normally distributed and nonparametric data are presented as means \pm SD and medians (ranges). Patient and nutritional characteristics were compared between groups with $t$ test, Mann-Whitney $U$ test, $\chi^{2}$ test, or Fisher's exact test for continuous normally distributed, nonparametric continuous, and dichotomous data, respectively. In the primary analysis, generalized estimating equations (GEE) were used to compare changes in f-IL-8 and f-calprotectin over time between the groups. This method takes into account the dependency of the observations within a patient and the fact that samples may not be available at each time point (24). In the crude GEE analysis, the first follow-up measurement was adjusted for the baseline value. For the remaining follow-up measurements, no adjustment was made (25). In additional GEE analyses, adjustments were made for chorioamnionitis, GA, BW, mode of delivery, Apgar score at $5 \mathrm{~min}<6$, exclusively breast milk fed, stay at a NICU or a general hospital ward, serious endogenous infection, postnatal antibiotic treatment (within $48 \mathrm{~h}$ preceding the sample), and NEC.

GEE analyses were also used to analyze the effect of perinatal factors on the development of f-IL- 8 and f-calprotectin over time. A Pearson correlation analysis was performed to analyze the correlation between f-IL- 8 and f-calprotectin. When the outcome variable was not normally distributed, a natural logarithm transformation was performed. All statistical analyses were performed on an intention to treat basis. A two-tailed $p$ value of $<0.05$ was considered significant, and SPSS 17.0 (SPSS Inc, Chicago, IL, USA) was used for data analysis.

\section{RESULTS}

Between May 2007 and November 2008, 113 of 208 eligible preterm infants entered the study. Reasons for not participating in the study were no informed consent $(n=45)$, participation in another trial $(n=7)$, transfer to a regional hospital within $48 \mathrm{~h}(n=12)$, death within $48 \mathrm{~h}(n=5)$, and severe congenital malformations $(n=12)$. After randomization, one infant in the placebo group was excluded, because of strong suspicion of a syndrome. Baseline patient and nutritional characteristics were not different in the ${ }_{\mathrm{SC}} \mathrm{GOS} / \mathrm{LC} \mathrm{FOS} /$ AOS group $(n=55)$ and placebo group $(n=58)$. Fecal samples for calprotectin and IL-8 were collected at $43.1 \pm$ $26.6 \mathrm{~h}(t=0)$ after birth, at postnatal d $7.5 \pm 1.0(t=1)$, $14.2 \pm 0.9(t=2)$, and $30.0 \pm 0.7(t=3)$. In total, $9 \%$ of the fecal samples were missing, mainly due to death, exclusion before the end of the study period, or no fecal sample available at that time point. F-IL-8 and f-calprotectin were correlated at all timepoints $\left(r^{2}=0.07 ; p<0.001\right)$. Analysis by GEE showed no effect of enteral supplementation of prebiotics on f-IL-8 and f-calprotectin (Fig. 1). In additional analyses, we found that adjustment for chorioamnionitis, GA, BW, mode of delivery, Apgar score at $5 \mathrm{~min}<6$, exclusively breast milk fed, stay at a NICU or a general hospital ward, serious endogenous infection and postnatal antibiotic treatment, and NEC did not change the primary results of the study (data not shown). As the results in the prebiotics group and the placebo group were not different, data of both groups were combined to analyze factors that may affect f-IL-8 and f-calprotectin. In total, 16 infants developed NEC, at a mean postnatal age of 28.8 ( \pm 16.0$)$ d. Infants who developed NEC had significantly increased levels of both f-IL-8 and f-Calprotectin compared with infants without NEC. Exclusive breast milk feeding during the study period coincides with an increase in f-IL-8 and f-calprotectin levels. Lower GA and lower BW increased f-IL-8 levels. Endogenous infections increased only f-IL-8 levels (Table 1).

\section{DISCUSSION}

In this study, enteral supplementation of ${ }_{\mathrm{SC}} \mathrm{GOS} /{ }_{\mathrm{LC}} \mathrm{FOS} /$ AOS between $\mathrm{d} 3$ and 30 of life does not affect the postnatal developmental pattern of f-IL-8 and f-calprotectin. Therefore, the positive effect of enteral supplementation of ${ }_{\mathrm{SC}} \mathrm{GOS} /$ ${ }_{\mathrm{LC}} \mathrm{FOS} / \mathrm{AOS}$ on serious infectious morbidity is not directly associated with f-IL-8 and f-calprotectin. In healthy term 

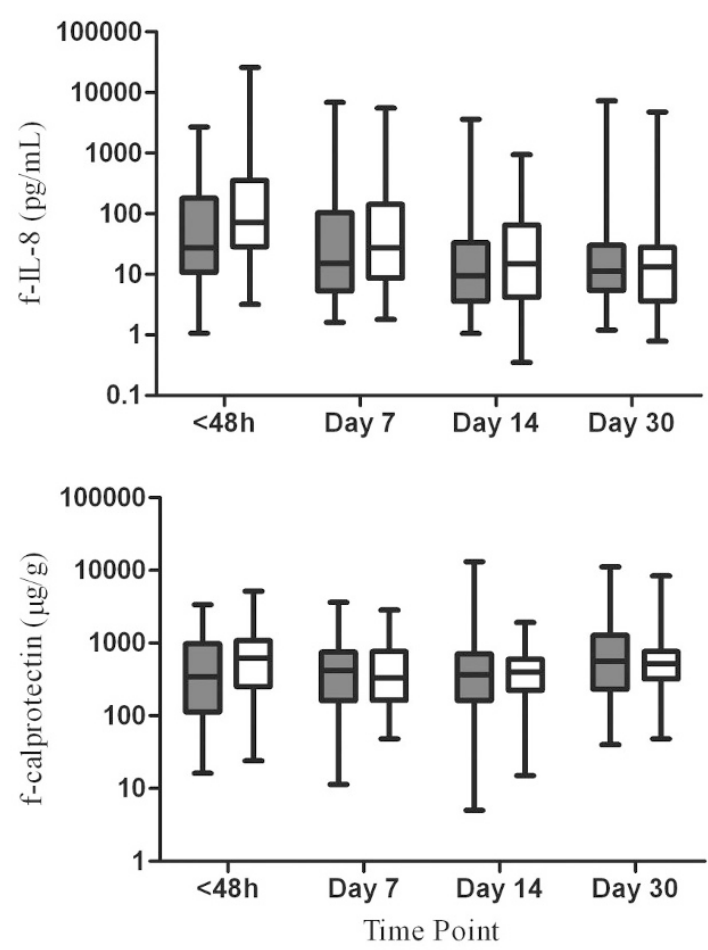

Figure 1. Effect of prebiotic mixture on f-IL-8 and f-calprotectin in preterm infants. Enteral supplementation of the prebiotic mixture consisting of neutral and acidic oligosaccharides had no effect on f-IL-8 and f-calprotectin as analyzed by GEE. Fecal samples were available for analysis of f-IL-8 and f-calprotectin at $t=0(n=102$ for both), $t=1(n=104$ and $n=103$, respectively), $t=2$ ( $n=105$ for both), and $t=3(n=98$ and $n=100$, respectively). Prebiotic mixture, $\mathbf{\square}$; placebo, $\square$. Bars indicate box plots with median and range.

Table 1. Factors influencing f-calprotectin and $f$-IL-8

\begin{tabular}{lcc}
\hline & \multicolumn{1}{c}{ F-IL-8 } & F-calprotectin \\
\hline Choroamnionitis & $1.36(0.77-2.43)$ & $1.06(0.79-1.42)$ \\
Antenatal coriticosteroids & $0.86(0.53-1.40)$ & $0.90(0.70-1.17)$ \\
GA (wk) & $0.72(0.65-0.81)^{*}$ & $1.05(0.99-1.12)$ \\
Birth weight (kg) & $0.23(0.12-0.42)^{*}$ & $1.00(0.64-1.52)$ \\
Vaginal delivery & $1.25(0.78-2.01)$ & $0.92(0.71-1.21)$ \\
Apgar score at 5 min <6 & $0.52(0.23-1.18)$ & $0.87(0.60-1.26)$ \\
Exclusive breast milk feeding & $1.59(0.99-2.54) \dagger$ & $1.47(1.10-1.98) \dagger$ \\
Necrotizing enterocolitis & $10.88(2.32-51.00) \ddagger$ & $5.17(1.76-15.16) \ddagger$ \\
Serious endogenous & $3.23(1.70-2.56)^{*}$ & $1.03(0.72-1.47)$ \\
$\quad$ infection§ & & \\
\hline
\end{tabular}

Data indicate the effect of a factor on f-IL-8 and f-calprotectin (GEE). The effect can be interpreted as follows: in case of chorioamnionitis, f-IL-8 is 1.36 $(95 \% \mathrm{CI})$ times as high as without chorioamnionitis.

$* p<0.001$.

$\dagger p<0.05$.

$\ddagger p<0.01$.

$\S$ Sepsis, meningitis, pyelonephritis, pneumonia, or arthritis as diagnosed by a combination of clinical signs and a positive culture with bacteria other than Coagulase Negative Staphylococci.

infants, Campeotto et al. (13) did not find an effect of prebiotics on f-calprotectin either.

During the study period, we found higher levels of f-calprotectin in preterm infants compared with the levels found in healthy children and adults (26). This is in line with other studies $(10,11,13)$. Jaarsma et al. (27) found an increased systemic inflammatory response in preterm infants directly after birth, and we hypothesize that the developing gastrointestinal tract is involved in this process. Furthermore, increased levels of f-IL-8 and f-calprotectin reflect the increase of neutrophils and monocytes in the intestinal lumen and could partly be explained by increased intestinal permeability in preterm infants (28).

Increased levels of f-IL-8 and f-calprotectin may also reflect the developing immune system. Acidic oligosaccharides may directly affect the immune cells via interaction of selectins, dendritic cell specific C-type lectin, integrins, and other target receptors as Toll-like receptors (15). Therefore, we hypothesize that increased levels of f-IL- 8 and f-calprotectin in the first period of life may be a reflection of a physiological maturation of the immune system and may not necessarily be harmful. Breast milk feeding may stimulate this maturation process of the immune system. We found increased levels of f-IL-8 and f-calprotectin in preterm infants who were fed exclusively on breast milk. For f-calprotectin, our results are in line with a recent study done by Savino et al. (19), who found higher levels of calprotectin in breastfed term infants compared with formula-fed term infants. The intestinal microbiota may play a role in this process (2). In infants with colic's, Rhoads et al. (29) found altered intestinal microbiota and increased calprotectin levels. The intestinal microbiota may stimulate transepithelial migration of granulocytes through chemotactic agents (30). Antibiotics delay the intestinal colonization in preterm infants (31). We hypothesize that delayed intestinal bacterial colonization, especially with "health promoting" bacteria, such as Bifidobacteria and (excessive) use of broad spectrum antibiotics contribute to sustained higher levels of f-IL-8 and f-calprotectin in preterm infants. In the immature gastrointestinal tract of these preterm infants, f-calprotectin may protect against inflammation through its antimicrobial and antifungal effects (8). Furthermore, both f-IL-8 and f-calprotectin may participate in the regulation of the intestinal bacterial colonization of the immature gut and may inhibit potential pathogenic bacterial growth.

Factors influencing f-IL-8 and f-calprotectin are not yet fully understood. In this study, levels of f-IL-8 were higher in infants with an endogenous infection than in infants without infection. Both f-IL-8 and f-calprotectin levels were higher in infants with NEC compared with either infants without NEC or infants with an endogenous infection. However, the levels of f-IL-8 and f-calprotectin were more increased in preterm infants with NEC than in infants with an endogenous infection. Therefore, we hypothesize that f-IL-8 and f-calprotectin may be used as noninvasive clinical markers for screening and follow-up of serious neonatal gastrointestinal disease. This hypothesis is supported by the findings of Yang et al. (17), who reported that $\mathrm{f}$-calprotectin in VLBW infants was higher $(>350 \mu \mathrm{g} / \mathrm{g})$ during major gastrointestinal manifestations (e.g. blood in stools, bowel perforation, distended abdomen with hypotension, and upper gastrointestinal contrast study) compared with minor infection or stress (e.g. urinary tract infection, feeding intolerance, and postpatent ductus arteriosus ligation). Larger studies of these markers are necessary to confirm clinical utility. The effect of GA, BW, and exclusive breast milk 
feeding on f-IL-8 and the effect of exclusive breast milk feeding on f-calprotectin may limit the usefulness of these markers of intestinal inflammation in this population. Nevertheless, serial measurements of f-IL-8 and f-calprotectin may be useful as a marker of the development of NEC. However, careful interpretation of the levels of f-IL-8 and f-calprotectin in the context of the clinical setting is warranted.

Some remarks may be formulated with regard to the methodology of our study. First, this is the first study measuring IL-8 in the feces of preterm infants and no comparison can be made with the literature. We found a correlation between f-IL-8 and f-calprotectin and, therefore, assumed that f-IL-8 may be a reliable marker for measuring intestinal inflammation. However, more studies are needed to determine the exact role of f-IL-8 in relation to intestinal inflammation. Second, the feces was collected from the diaper instead of direct collection, which may have increased the concentrations of f-calprotectin and f-IL-8 by absorption of water by the diaper. Olafsdottir et al. (32) showed that collection of feces from diapers increase f-calprotectin concentrations with $30 \%$ compared with directly collected feces. Although this effect may also apply to this study, all fecal samples have been collected in the same way and this will not influence the results of our study. Third, breast milk itself contains neutral and acidic oligosaccharides. Therefore, the effect of enteral supplementation of prebiotics may be less pronounced in preterm infants who received breast milk exclusively. However, adjusting the analysis for type of feeding did not change the results of the crude analysis. As breast milk is strongly promoted at our NICU, most infants received breast milk $(>60 \%)$, and relatively few received exclusive formula feeding (20\%). Fourth, although this particular formulation of prebiotic oligosaccharides did not affect the levels of f-IL-8 and f-calprotectin, this does not preclude the possibility that other formulation may do so.

To conclude, we found that enteral supplementation of a prebiotic mixture consisting of neutral and acidic oligosaccharides does not affect f-IL-8 and f-calprotectin levels in preterm infants. In a previous study, we found that if ${ }_{\mathrm{SC}} \mathrm{GOS} / \mathrm{LC} \mathrm{FOS} /$ AOS was given in sufficient amounts (per-protocol analysis), there was a trend toward a lower incidence of serious endogenous infections in preterm infants. This decrease of serious endogenous infections is not directly related to a lower intestinal inflammatory response as measured by f-IL-8 and f-calprotectin. However, the intestinal inflammatory response may still play a role in the susceptibility to (endogenous) infections. Furthermore, other factors, such as increased intestinal colonization with a "bifidogenic" microbiota, may play a role in the beneficial effect of neutral and acidic ligosaccharides in preterm infants.

Acknowledgments. We are indebted to the parents for allowing their infants to participate in the study. Furthermore, we thank the medical and nursing staff of the neonatal intensive care unit of the VU University Medical Center and of all participating hospitals.

\section{REFERENCES}

1. Field CJ 2005 The immunological components of human milk and their effect on immune development in infants. J Nutr 135:1-4
2. Lu L, Walker WA 2001 Pathologic and physiologic interactions of bacteria with the gastrointestinal epithelium. Am J Clin Nutr 73:1124S-1130S

3. Claud EC, Walker WA 2001 Hypothesis: inappropriate colonization of the premature intestine can cause neonatal necrotizing enterocolitis. FASEB J 15:1398-1403

4. Mitsuyama K, Toyonaga A, Sasaki E, Watanabe K, Tateishi H, Nishiyama T, Saiki T, Ikeda H, Tsuruta O, Tanikawa K 1994 IL-8 as an important chemoattractant for neutrophils in ulcerative colitis and Crohn's disease. Clin Exp Immunol 96:432-436

5. Reddy KP, Markowitz JE, Ruchelli ED, Baldassano RN, Brown KA 2007 Lamina propria and circulating interleukin- 8 in newly and previously diagnosed pediatric inflammatory bowel disease patients. Dig Dis Sci 52:365-372

6. Nanthakumar NN, Fusunyan RD, Sanderson I, Walker WA 2000 Inflammation in the developing human intestine: a possible pathophysiologic contribution to necrotizing enterocolitis. Proc Natl Acad Sci USA 97:6043-6048

7. Zali H, Rezaei-Tavirani M, Kariminia A, Yousefi R, Shokrgozar MA 2008 Evaluation of growth inhibitory and apoptosis inducing activity of human calprotectin on the human gastric cell line (AGS). Iran Biomed J 12:7-14

8. Steinbakk M, Naess-Andresen CF, Lingaas E, Dale I, Brandtzaeg P, Fagerhol MK 1990 Antimicrobial actions of calcium binding leucocyte L1 protein, calprotectin. Lancet 336:763-765

9. Campeotto F, Baldassarre M, Butel MJ, Viallon V, Nganzali F, Soulaines P, Kalach N, Lapillonne A, Laforgia N, Moriette G, Dupont C, Kapel N 2009 Fecal calprotectin: cutoff values for identifying intestinal distress in preterm infants. J Pediatr Gastroenterol Nutr 48:507-510

10. Josefsson S, Bunn SK, Domellof M 2007 Fecal calprotectin in very low birth weight infants. J Pediatr Gastroenterol Nutr 44:407-413

11. Nissen AC, van Gils CE, Menheere PP, Van den Neucker AM, van der Hoeven MA, Forget PP 2004 Fecal calprotectin in healthy term and preterm infants. J Pediatr Gastroenterol Nutr 38:107-108

12. Carroll D, Corfield A, Spicer R, Cairns P 2003 Faecal calprotectin concentrations and diagnosis of necrotising enterocolitis. Lancet 361:310-311

13. Campeotto F, Butel MJ, Kalach N, Derrieux S, Aubert-Jacquin C, Barbot L, Francoual C, Dupont C, Kapel N 2004 High faecal calprotectin concentrations in newborn infants. Arch Dis Child Fetal Neonatal Ed 89:F353-F355

14. Bode L 2006 Recent advances on structure, metabolism, and function of human milk oligosaccharides. J Nutr 136:2127-2130

15. Boehm G, Moro G 2008 Structural and functional aspects of prebiotics used in infant nutrition. J Nutr 138:1818S-1828S

16. Niñonuevo MR, Lebrilla CB 2009 Mass spectrometric methods for analysis of oligosaccharides in human milk. Nutr Rev 67:S216-S226

17. Yang Q, Smith PB, Goldberg RN, Cotten CM 2008 Dynamic change of fecal calprotectin in very low birth weight infants during the first month of life. Neonatology 94:267-271

18. Dorosko SM, Mackenzie T, Connor RI 2008 Fecal calprotectin concentrations are higher in exclusively breastfed infants compared to those who are mixed-fed. Breastfeed Med 3:117-119

19. Savino F, Castagno E, Calabrese R, Viola S, Oggero R, Miniero R 2010 High faecal calprotectin levels in healthy, exclusively breast-fed infants. Neonatology 97:299-304

20. Baldassarre ME, Altomare MA, Fanelli M, Carbone D, Di Bitonto G, Mautone A, Laforgia N 2007 Does calprotectin represent a regulatory factor in host defense or a drug target in inflammatory disease? Endocr Metab Immune Disord Drug Targets 7:1-5

21. Westerbeek EA, van den Berg JP, Lafeber HN, Fetter WP, Boehm G, Twisk JW, van Elburg RM 2010 Neutral and acidic oligosaccharides in preterm infants: a randomized, double-blind, placebo-controlled trial. Am J Clin Nutr 91:679-686

22. Westerbeek EA, van Elburg RM, van den Berg A, van den Berg J, Twisk JW, Fetter WP, Lafeber HN 2008 Design of a randomised controlled trial on immune effects of acidic and neutral oligosaccharides in the nutrition of preterm infants: carrot study. BMC Pediatr 8:46

23. Bell MJ, Ternberg JL, Feigin RD, Keating JP, Marshall R, Barton L, Brotherton T 1978 Neonatal necrotizing enterocolitis. Therapeutic decisions based upon clinical staging. Ann Surg 187:1-7

24. Twisk JW, Smidt N, de Vente W 2005 Applied analysis of recurrent events: a practical overview. J Epidemiol Community Health 59:706-710

25. Twisk JW, de Vente W 2008 The analysis of randomised controlled trial data with more than one follow-up measurement. A comparison between different approaches. Eur J Epidemiol 23:655-660

26. Konikoff MR, Denson LA 2006 Role of fecal calprotectin as a biomarker of intestinal inflammation in inflammatory bowel disease. Inflamm Bowel Dis 12:524534

27. Jaarsma AS, Braaksma MA, Geven WB, van Oeveren W, Bambang Oetomo S 2004 Activation of the inflammatory reaction within minutes after birth in ventilated preterm lambs with neonatal respiratory distress syndrome. Biol Neonate 86:1-5

28. Berstad A, Arslan G, Folvik G 2000 Relationship between intestinal permeability and calprotectin concentration in gut lavage fluid. Scand J Gastroenterol 35:64-69

29. Rhoads JM, Fatheree NY, Norori J, Liu Y, Lucke JF, Tyson JE, Ferris MJ 2009 Altered fecal microflora and increased fecal calprotectin in infants with colic. J Pediatr 155:823.e1-828.e1

30. Nash S, Stafford J, Madara JL 1987 Effects of polymorphonuclear leukocyte transmigration on the barrier function of cultured intestinal epithelial monolayers. J Clin Invest 80:1104-1113

31. Westerbeek EA, van den Berg A, Lafeber HN, Knol J, Fetter WP, van Elburg RM 2006 The intestinal bacterial colonisation in preterm infants: a review of the literature. Clin Nutr 25:361-368

32. Olafsdottir E, Aksnes L, Fluge G, Berstad A 2002 Faecal calprotectin levels in infants with infantile colic, healthy infants, children with inflammatory bowel disease, children with recurrent abdominal pain and healthy children. Acta Paediatr 91:45-50 